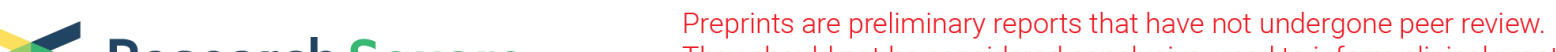 Research Square They should not be considered conclusive, used to inform clinical practice, or referenced by the media as validated information.
}

\section{Clinical And Epidemiologic Profile of Breast Cancer Differences In Mexico: A Retrospective Cohort Study From Guerrero.}

\section{Azucena Ocampo-Bárcenas}

Instituto de Cancerologia

Marlon De Ita Ley

Instituto Mexicano del Seguro Social

Martín Morrugares-Ixtepan

Instituto de Cancerologia

Abril Bautista-Escutia

Instituto de Cancerologia

Ricardo Hernández-Morales

Instituto de Cancerologia

Eloisa Ibarra Sierra

Instituto de Cancerologia

Marco Antonio Jiménez-López ( $\nabla$ marcoajlpatologia@gmail.com )

Instituto de Cancerología: Instituto de Cancerologia https://orcid.org/0000-0002-9712-9577

\section{Research Article}

Keywords: Breast cancer, Mexico, Regions, Survival time, Molecular subtype

Posted Date: June 24th, 2021

DOl: https://doi.org/10.21203/rs.3.rs-575884/v1

License: (c) (i) This work is licensed under a Creative Commons Attribution 4.0 International License. Read Full License 


\section{Abstract}

Background: The regional disparities in Mexico impact the incidence and mortality of breast cancer (BC). Despite Guerrero state has a high grade of economic marginalization, it presents mortality lower than the national average. Clinical, pathological, and epidemiological assessment will help to understand the source of these differences.

Objective: Describe the clinical, pathological, and overall survival of patients with BC from Guerrero and compare them with other reports from the Mexican population.

Materials and Methods: Kaplan-Meier method was used to estimate overall survival time over a retrospective cohort of breast cancer patients treated at the Instituto Estatal de Cancerología "Dr. Arturo Beltrán Ortega (IECan) during 2010-2020. The Cox proportional hazards model was used to determine the prognosis factors.

Results: A total of 923 women were included. $3.5 \%$ and $37.9 \%$ of patients were classified as Stage I and II; for locally advanced stages III and IV, it has been observed $36.1 \%$ and $14.3 \%$ of patients respectively. The most frequent histological type was ductal carcinoma (82.7\%). The molecular subtype percentage found were HR+/HER2- (35.1\%), HR \pm /HER2- (15.9\%), HR \pm /HER2+ (14.4\%), HR-/HER2 + (13.5\%) and HR-/HER2 $(21 \%)$. The median follow-up was 49 months. Five-year survival for the entire cohort was $78 \%(95 \% \mathrm{Cl}, 73-$ $82 \%)$, and $31 \%$ for metastatic disease $(95 \% \mathrm{Cl}, 26.7-37.1 \%)$.

Conclusion: The Guerrero state shares epidemiological parameters with other regions of Mexico; despite this, presents frequently one of the most aggressive molecular subtypes of breast cancer; so, the understanding of its lower mortality requires further analysis.

\section{Introduction}

Breast cancer $(\mathrm{BC})$ is the most common malignant tumor in women worldwide and represents the leading cause of death from cancer (1). Despite the advances in diagnosis and treatment, $45 \%$ of the global incidence occurs in developing countries, representing 55\% of all deaths. In Mexico, Globocan estimates that BC incidence and mortality is 39.5 and 9.9 cases per 100,000, respectively (2). Besides, the Instituto Nacional de Cancerología (National Cancer Institute, INCan) and Fundación de Cancer de Mama (Breast Cancer Foundation, FUCAM), two reference institutions in BC in our country have taken the task of describing accurately the clinical and epidemiological characteristics in the Mexican population $(3,4)$. Despite these institutions concentrate information from patients of different geographical regions, is still necessary to explore the behavior of this disease locally, especially in regions where the ethnic origin is diverse, social and economic marginalization presents a health challenge, or the local health infrastructure presents issues in the care of BC patients (4).

In Mexico, the biological, social, and cultural disparities between populations' have an impact on the BC incidence and mortality; it has been reported epidemiological differences between the north, center, and 
southeast geographic regions; especially in the last one which presents high marginalization but one of the lowest mortality rate in the country (5). Today, local and regional epidemiological information is essential, the BC standard management considers broad aspects, including epidemiological, clinical, and anatomopathological factors; this information allows to stratify patients into BC risk groups to treat and follow them; so, identifying each local population characteristics will allow a better approach from preventive medicine $(3,6,7)$. To understand the mortality rate differences observed in national studies, this study focuses on describing the clinical, pathological, and epidemiological characteristics of breast cancer patients from Guerrero and compares them with other reports from the Mexican population.

\section{Materials And Methods}

A retrospective cohort study of breast cancer patients treated at the Instituto Estatal de Cancerología "Dr. Arturo Beltran Ortega" (IECan) from Acapulco, Guerrero, Mexico was conducted. This study was approved by the Research Ethics Committee; the study was considered minimal risk; so, informed consent was not required. A total of 923 medical records of patients were reviewed, patients who underwent diagnosis/treatment within the institute, whose follow-up was 10 years, and were covered by the Fondo de Protección contra Gastos Catastróficos (Catastrophic Expenses Protection Fund) were included. The clinicopathological characteristics, treatment, histopathological type, immunohistochemistry (IHC) profile, and current patient status were analyzed. Histopathologic evaluations were performed by pathologists; the presence of hormone receptors (HR) was determined by IHC using the H-Score (5). A score greater than 10 (more than $10 \%$ positive cells) was used to define estrogen receptor (ER) and progesterone receptor (PR) positivity. HER2 status was determined by IHC; those samples identified as zero (no membrane staining) or 1+ (weak and incomplete staining) and 2+ (nonspecific) or negative Fluorescent In Situ Hybridization (FISH) were considered negative. Tumors were considered HER2 + if they were classified as $3+$ on membrane staining or amplified FISH. According to the $\mathrm{IHC}$ results the samples were classified as luminal $A(R H+/ H E R-)$, luminal $B(R H \pm / H E R 2-$ and $R H \pm /$ HER2+), HER2 positive (RH-/HER2+), triple-negative (RH-/HER2-). The clinical staging of each patient was established by standardized universal criteria, using the TNM system of the American Joint Committee on Cancer (AJCC), based on tumor size, lymph node status, and metastasis. The Scarff-Bloom-Richardson (SBR) histological grade was used to establish the degree of malignancy.

\section{Statistical analysis}

Risk factors were categorized based on those described in various epidemiological studies of breast cancer, overall survival (OS), and Disease-free survival (DFS) was determined using the Kaplan-Meier method. Statistical differences between survival functions for clinical characteristics were calculated using the Log-Rank or Wilcoxon test. An adjusted Cox proportional hazards model was used to identify the clinical variables predictive of survival in the study population. A $p \leq 0.05$ was considered statistically significant. All analyzes were performed in SPSS v.25. 


\section{Results}

The study reviewed and analyzed 923 medical records of patients diagnosed with breast cancer from January 2010 to December 2020. The mean age at the time of diagnosis was 53.0 with an SD of \pm 12.0 , with an age range from 19 to 93 years old. The population consisted of $91.3 \%$ women from the 7 regions of the state of Guerrero (Supplementary 1), the remaining 8.7\% corresponded to patients from neighboring states such as Michoacán, Morelos, and Oaxaca, as well as native women from other states, who reside permanently in Guerrero; despite this, all patients were included in the study (Suplemmentary).

The principal comorbidities observed were type II diabetes at 7.5\%; hypertension at $7.2 \%$; type II diabetes/hypertension at $14.7 \%$ and others at $2.1 \%$ of patients. Related to familiar cancer history, $17.2 \%$ of the population presented a first-degree family with a cancer diagnosis. We have also observed that the average age of menarche is $12.9 \pm 1.3$ years, the first pregnancy was at $21.6 \pm 5.2$ years, while menopause was $45.7 \pm 5.1$ years: in $236(25.6 \%)$ patients reported the use of contraceptives (Table 1 ). 
Table 1

Description of demographic and reproductive factors in BC patients.

\begin{tabular}{|c|c|c|}
\hline \multicolumn{2}{|l|}{ Characteristic } & Frequency (\%) \\
\hline \multicolumn{2}{|l|}{ Age, years $($ mean $\pm S D)$} & $53.0 \pm 12.0$ \\
\hline \multicolumn{2}{|l|}{ Median (Rank), years } & $52(19-93)$ \\
\hline \multirow[t]{2}{*}{ Family history of cancer } & No & $764(82.8)$ \\
\hline & Yes & $159(17.2)$ \\
\hline \multirow[t]{4}{*}{ Comorbidity } & Diabetes & $64(6.9)$ \\
\hline & Hypertension & $137(14.8)$ \\
\hline & Diabetes and Hypertension & $65(7.0)$ \\
\hline & Other & $13(1.4)$ \\
\hline \multirow[t]{10}{*}{ Reproductive factors } & Age at menarche, years ( mean $\pm S D$ ) & $12.9 \pm 1.3$ \\
\hline & Median (Rank), years & $13(9-17)$ \\
\hline & Age first pregnancy, years (mean $\pm S D$ ) & $21.6 \pm 5.2$ \\
\hline & Median (Rank), years & $20(13-45)$ \\
\hline & Oral contraceptive use & $236(25.6)$ \\
\hline & Age at menopause, years (mean \pm SD) & $45.7 \pm 5.1$ \\
\hline & Median (Rank), years & $47(23-63)$ \\
\hline & \multicolumn{2}{|l|}{ Menopausal status } \\
\hline & $\leq 50$ & $590(64)$ \\
\hline & $>50$ & $89(10)$ \\
\hline
\end{tabular}

Regarding the diagnosis of breast cancer, it has been observed that $41.4 \%$ of patients were in stage I and II clinical stages at the time of diagnosis, $3.5 \%$ in stage I and $37.9 \%$ for stage II; for locally advanced stages III and IV, it has been observed $36.1 \%$ and $14.3 \%$ of patients respectively, for the rest $8.2 \%$ there was insufficient information to classify the carcinoma. Concerning to histological type of BC, $82.7 \%$ of patients presented ductal carcinoma (Table 2). When the tumor was segmented by histological grade, of the total, $7.2 \%$ corresponded well-differentiated tumors or grade I, $45.9 \%$ moderately differentiated (grade II) and $40.6 \%$ poorly differentiated (grade III). The molecular subtype most common was HR+/HER2$(35.1 \%, n=324)$, HR \pm /HER2- $(15.9 \%, n=147)$, HR \pm /HER2 $+(14.4 \%, n=133)$, HR-/HER2+ $(13.5 \%, n=125)$ and HR-/HER2- $(21 \%, n=194)$ (Table 2$)$. 
Table 2

Clínical and pathological characteristics of BC Patients.

\begin{tabular}{|c|c|c|}
\hline Variable & Characteristic & Frequency (\%) \\
\hline \multirow[t]{3}{*}{ Histopathology } & Ductal & 763 (82.7) \\
\hline & Lobular & $38(4.1)$ \\
\hline & Other & $122(13.2)$ \\
\hline \multirow[t]{3}{*}{ Tumor size } & $\leq 2 \mathrm{~cm}$ & 431 (46.7) \\
\hline & $2-5 \mathrm{~cm}$ & $361(39.1)$ \\
\hline & $>5 \mathrm{~cm}$ & $54(5.9)$ \\
\hline \multirow[t]{3}{*}{ Histologic grade } & Low & $66(7.2)$ \\
\hline & Intermediate & $424(45.9)$ \\
\hline & High & $402(43.6)$ \\
\hline \multirow[t]{2}{*}{ Lymph node metastasis } & Positive & $467(51)$ \\
\hline & Negative & $432(47)$ \\
\hline \multirow[t]{4}{*}{ Clinical stage } & I & $32(3.5)$ \\
\hline & II & 350 (37.9) \\
\hline & III & $333(36.1)$ \\
\hline & IV & $132(14.3)$ \\
\hline \multirow[t]{5}{*}{ Molecular subtype } & HR+/HER2NEU- & $324(35.1)$ \\
\hline & HR $\pm / H E R 2 N E U-$ & $147(15.9)$ \\
\hline & HR $\pm / H E R 2 N E U+$ & $133(14.4)$ \\
\hline & HR-/HER2NEU+ & $125(13.5)$ \\
\hline & HR-/HER2NEU- & $194(21)$ \\
\hline \multirow[t]{2}{*}{ Surgical treatment } & Mastectomy & 894 (96.9) \\
\hline & Breast-conserving surgery & $29(3.1)$ \\
\hline \multirow[t]{2}{*}{ Chemotherapy } & Adjuvant & $469(49.8)$ \\
\hline & Neoadjuvant & $363(39.4)$ \\
\hline \multirow[t]{2}{*}{ Radioteraphy } & Yes & $557(60.3)$ \\
\hline & No & 366 (39.6) \\
\hline
\end{tabular}


Related to treatment, $96.9 \%$ of the population underwent a mastectomy, $89.2 \%$ received chemotherapy, from which $49.8 \%$ were adjuvant and $39.4 \%$ were neoadjuvant, while $60.3 \%$ of the population received radiotherapy (Table 2). Median follow-up for the entire cohort was 49 months (Q1-Q3, 31 to 69 mo.). Thirty-one percent of patients $(n=290)$ presented metastatic disease, and $24.6 \%(n=227)$ died. The median overall survival (OS) was 93 months ( $95 \%$ Cl 80.48-105.51 mo.). The 5-year OS was 78.0 months (95\% Cl, 73.01-82.98 mo.), and for those with metastatic disease, it was 31.91 months ( $95 \% \mathrm{Cl}, 26.70$ 37.13 mo.). Survival curves according to demographic and clinical characteristics can be seen in Fig. 1. Multivariate analysis showed that survival is associated with age at the time of diagnosis, it is significantly lower in patients aged $\leq 40$ years, histological grade 3 , advanced stage of diagnosis, tumor size, and HR \pm /HER2+, HR-/HER2+, HR-/HER2- tumors (Table 3).

Table 3

Multivariate analysis for breast cancer-specific survival (Cox proportional regression model)

\begin{tabular}{|c|c|c|c|c|}
\hline \multirow{2}{*}{ Characteristic } & & \multicolumn{3}{|c|}{ Multivariate analysis } \\
\hline & & $H R$ & $(95 \% \mathrm{Cl})$ & $p$ value \\
\hline \multicolumn{5}{|l|}{ Age, years } \\
\hline$\leq 40$ & & - & - & - \\
\hline$>40$ & & 2.090 & $1.56-2.81$ & $<0.001$ \\
\hline \multicolumn{5}{|l|}{ Clinical stage } \\
\hline I & & - & - & - \\
\hline II & & 0.273 & $0.059-1.256$ & 0.095 \\
\hline III & & 0.327 & $0.071-1.512$ & 0.153 \\
\hline IV & & 0.487 & $0.103-2.288$ & 0.362 \\
\hline \multicolumn{5}{|c|}{ Breast cancer subtype } \\
\hline HR+/HER2- & Luminal A & 0.38 & $0.26-0.56$ & $<0.001$ \\
\hline HR $\pm / H E R 2-$ & Luminal B & 0.36 & $0.22-0.59$ & $<0.001$ \\
\hline HR $\pm / H E R 2+$ & Luminal B & 0.68 & $0.45-1.02$ & 0.064 \\
\hline HR-/HER2+ & Her2 + & - & - & - \\
\hline HR-/HER2- & Triple negative & 1.06 & $0.73-1.52$ & 0.8 \\
\hline \multicolumn{5}{|l|}{ Metastasis } \\
\hline No & & - & - & - \\
\hline Yes & & 31.54 & $16.60-59.93$ & $<0.0001$ \\
\hline
\end{tabular}




\section{Discussion}

Breast cancer is the most common neoplasm in women in Mexico (5). INCan and FUCAM have taken on the task of describing the clinical-epidemiological characteristics of the populations they serve; despite this, it is still necessary to explore and understand the behavior of BC in other country regions (4). Three of the most marginalized states of México (Guerrero, Oaxaca, and Chiapas) are in the lowest BC mortality rate in the country (5). This mortality rate is still unexplained; therefore, we have analyzed several prognostic factors that could be related to this and compared them to other regions of the country. Reports from the states of Jalisco, Mexico City, and Veracruz have contributed significantly to the study of breast cancer epidemiology in our country, and the two first had one of the highest mortality rates in the country. The findings of the previous reports give an approximation of the heterogeneity of the disease and outcomes depending on the geographical region analyzed. One of the most relevant characteristics is the age at the time of diagnosis; Rodríguez et al. in 2017 described that in Mexico the mean is 53 years old, lower than reported of populations in developed countries (3). On the other hand, the clinical stage is another important characteristic in the Mexican population: diagnosed patients present more advanced stages, compared to the data published by countries that have early detection programs $(3,6)$. In Guerrero, the incidence by age group shows a behavior like that described by Rodríguez et al., the average age at diagnosis was 53 years, where the groups with the highest prevalence were 41 to 50 years and 51 to 60 years.

The histological grade is considered a determining factor of the biological behavior of the tumor and a useful prognostic tool. The population presented a greater number of cases with intermediate and high histological grade, both with a similar incidence, as reported by INCan, FUCAM, and the Breast Clinic of the Instituto Jalisciense de Cancerologia (Guadalajara, México) where $90 \%$ of the patients presented histological grade II and III $(2,4,8)$. Hormone receptors (estrogen and progesterone) and overexpression of the HER-2 oncoprotein are important prognostic and predictive factors (5). According to the 2019 Colima Consensus on Breast Cancer, the classification of the subtypes was established as Luminal A, Luminal B, HER-2, and Triple-negative, while their immunohistochemical approach was Luminal A (HR+/HER2-), Luminal B HR \pm /HER2- and HR \pm / HER2+), HER-2 (HR-/HER2+), and Triple-negative (HR-/HER2-) (5). Our subtypes analysis was based on the immunohistochemical approach since it gave us a broad overview of their behavior and allowed us to establish differences between them.

When we tried to contrast our results with data from other's institutions, as INCan, FUCAM, Jalisco, and Veracruz, we found that different workgroups disagree in the reporting of their results; so, to compare them, we standardized the data from different populations according to the Classification of Colima Consensus. In Guerrero, $65.4 \%$ of the tumors are of the luminal subtype (HR+/HER2-, HR \pm / HER2- and HR \pm /HER2+), like Veracruz (65.4\%), slightly different from Jalisco (68\%) and INCan (60.7\%), but not so for FUCAM (76.6\%). Regarding the HER2 + tumor subtype, Guerrero, showed a prevalence of $14 \%$, slightly higher than the $9 \%$ reported by FUCAM and the $11 \%$ in Jalisco and Veracruz, while we observed that HER2 + is more prevalent in INCan (23\%). Finally, the triple-negative subtype in Guerrero presented a prevalence of $21 \%$ equal to that reported by Jalisco and slightly lower than $23.5 \%$ of the cases in 
Veracruz. Interestingly, the prevalence of the triple-negative subtype in INCan (16\%) and FUCAM (15\%) is lower than the data previously cited $(2-4,9)$

Comparing our survival results, we found that the median follow-up was 49 months, higher than that reported by INCan (40.5 months), Jalisco ( 46.8 months), and FUCAM ( 28 months). The 5 -year overall survival in Guerrero was $78 \%$ like that reported by Jalisco (78.5\%), a percentage slightly lower than the survival reported by INCan (82\%) and FUCAM (83\%). Regarding survival by subtypes, Guerrero presented $80 \%$ survival for the luminal subtype (HR+/HER2 NEU- and HR $\pm /$ HER2-), lower than that reported by FUCAM and Jalisco, who reported $89 \%$. When these subtypes were individually analyzed, we found that in Guerrero the survival of patients with the HR+/HER2- subtype was $76 \%$ vs 84 of the patients who presented the HR $\pm / H E R 2-$ subtype. On the other hand, Guerrero and FUCAM presented the same survival percentage for the HR \pm /HER2 + subtype, while Jalisco reported $82 \%$. For HER2 + Guerrero showed a $70 \%$ survival, this data is like the $69 \%$ reported by the FUCAM. Finally, the survival for the triple-negative subtype in Guerrero was 73\%, the same as that reported by the FUCAM, while Jalisco reported a $70 \%$ survival rate (2-4). The differences in the tumor subtype in our population compared to populations from other states provides important data in the study of tumor heterogeneity.

Most of our patients were diagnosed with advanced-stage breast cancer, this fact can be attributed to the high rate of population marginalization. This condition is evidence of a low educational and health level, where the per capita income is one of the lowest in comparison with the rest of the states of Mexico(10). Other possible causes of these differences can be attributed to the influence of factors such as population aging, the "westernization" of the lifestyle, deficiencies in health education and information, as well as the genetic background of each population (5). Although several factors were included to identify regional differences that could explain the $\mathrm{BC}$ lower mortality found in Guererro, the results only display a relatively higher triple-negative frequency in this study in comparison to other reports, but we have not found a difference in survival between patients with this diagnosis. Currently, our country does not have studies that make a detailed description of this characteristic in a particular BC population; understanding the molecular and cellular mechanisms of tumor heterogeneity is relevant in the search for individualized treatments.

In conclusion, the characterization of the clinical-epidemiological profile of each region is essential in the identification of risk and prognostic factors, which are essential in the improvement of strategies aimed at decision making for individualized treatment. In the case of Guerrero, despite the marginalization, most of the epidemiological parameters are shared with other entities in various reports in the literature, only the frequency of the triple-negative being different in the population of this study. Therefore, the BC mortality rates differences are still unexplained and required further analysis; however, it is noted that it is necessary to follow a specific parameters to be able to report breast cancer cases at the national level to make appropriate comparisons at the national and local levels.

\section{Declarations}




\section{Conflict of Interest}

The authors declare that there are no conflicts of interest.

\section{Acknowledgments}

We express our gratitude to the participants of this study, as well the clinical archive staff at Instituto Estatal de Cancerología "Dr. Arturo Beltran Ortega".

\section{Corresponding author}

Correspondence Marco Antonio Jimenez Lopez

\section{References}

1. Sung H, Ferlay J, Siegel RL, Laversanne M, Soerjomataram I, Jemal A et al (2021) Global Cancer Statistics 2020: GLOBOCAN Estimates of Incidence and Mortality Worldwide for 36 Cancers in 185 Countries. CA Cancer J Clin 71(3):209-249

2. Dorado-Roncancio IF, V-NJJ, Hernández-Garibay AH (2020) t García González I.J. Breast cancer survival at 5 years: Experience of an institution at Jalisco, México. Ginecología y Obstetricia de México 88(5):9

3. Maffuz-Aziz A, Labastida-Almendaro S, Espejo-Fonseca A, Rodriguez-Cuevas S (2017) [Clinical and pathological features of breast cancer in a population of Mexico]. Cir Cir 85(3):201-207

4. Reynoso-Noveron N, Villarreal-Garza C, Soto-Perez-de-Celis E, Arce-Salinas C, Matus-Santos J, Ramirez-Ugalde MT et al (2017) Clinical and Epidemiological Profile of Breast Cancer in Mexico: Results of the Seguro Popular. J Glob Oncol 3(6):757-764

5. Cárdenas-Sánchez JEV-SAA, Arce-Salinas C, Bargalló-Rocha JE, Bautista-Piña V, Cervantes-Sánchez G, Flores-Balcázar C, Lluch-Hernández A, Maffuz-Aziz A, Pérez-Sánchez VM, Poitevin-Chacón A, Salas-González Torrecillas-Torres L., and Valero-Castillo V. Consenso Mexicano sobre diagnóstico y tratamiento del cáncer mamario. Octava revisión. Colima (2019) Gaceta Mexicana de Oncología. 2019;18:91

6. Maffuz-Aziz A, Labastida-Almendaro S, Sherwell-Cabello S, RuvalcabaLimon E, Dominguez-Reyes CA, Tenorio-Torres JA et al (2016) [Breast Cancer Survival: Clinical andPathological Prognostic Factors Analysis]. Ginecol Obstet Mex 84(8):498-506

7. Reyna-Sevilla A, GCME, Ramos Herrera IM (2016) Prevalencia de cáncer en Guerrero, México: un indicador para la prevención y el diagnóstico oportuno. SALUD JALISCO 1:7

8. Alarcon Rojas CA, Alvarez-Banuelos MT, Morales-Romero J, Suarez-Diaz H, Hernandez-Fonseca JC, Contreras-Alarcon G (2019) Breast Cancer: Metastasis, Molecular Subtypes, and Overweight and Obesity in Veracruz, Mexico. Clin Breast Cancer 19(1):e166-e171 
9. Cahua-Pablo JA, Cruz M, Tello-Almaguer PV, Del Alarcon-Romero LC, Parra EJ, Villerias-Salinas S et al. Analysis of admixture proportions in seven geographical regions of the state of Guerrero, Mexico. Am J Hum Biol. 2017;29(6)

10. R MH (2015) Análisis regional de la marginación en el estado de Guerrero, México. Papeles de población 21(84):24

\section{Figures}


A)

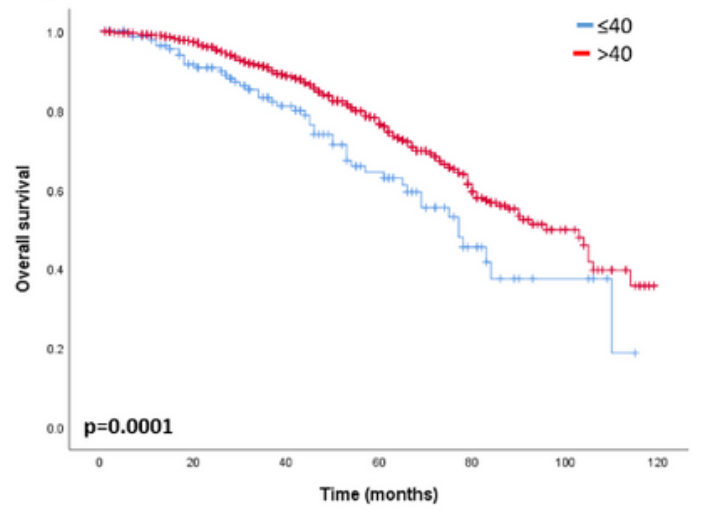

$\begin{array}{lllllll}\text { No. at risk (events) } & 0 & 20 & 40 & 60 & 80 & 100\end{array}$

$\leq 40 \quad 133(11) \quad 99(11) \quad 65(13) \quad 34(9) \quad 11(2) \quad 3(1)$

$>40$
B)

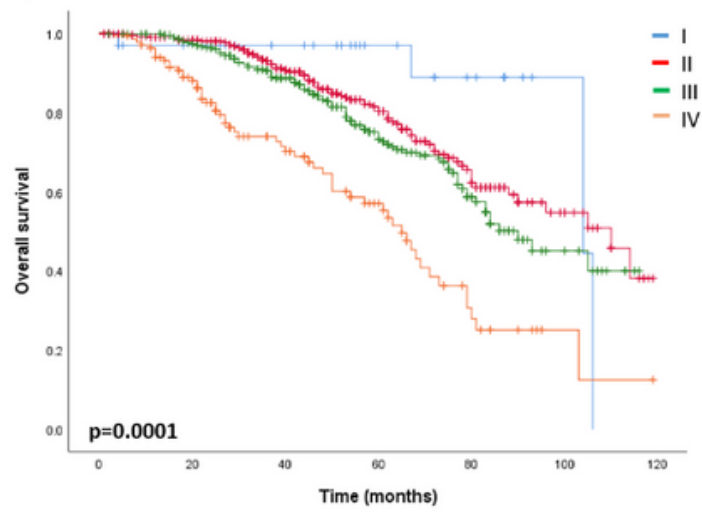

C)

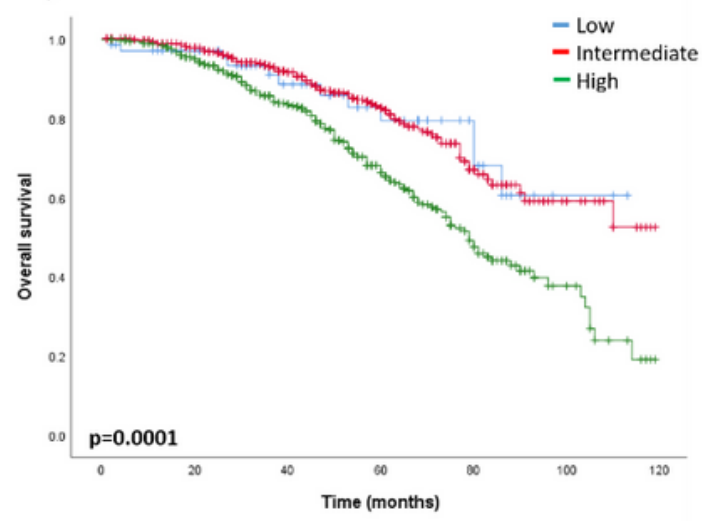

$\begin{array}{lllllll}\text { No. at risk (events) } & 0 & 20 & 40 & 60 & 80 & 100\end{array}$

Low $\quad 63(2) \quad 49(4) \quad 32(2) \quad 20(1) \quad 10(3) \quad 1(0)$

Intermediate

High

$63(2) \quad 49(4) \quad 32(2) \quad 20(1) \quad 10(3) \quad 1(0)$

$408(9) \quad 339(21) \quad 229(22) \quad 121(22) \quad 42(5) \quad 9(1)$

$403(19) 338(41) \quad 228(41) \quad 121(32) \quad 44(10) \quad 11(6)$

$\begin{array}{lcccccc}\text { No. at risk (events) } & 0 & 20 & 40 & 60 & 80 & 100 \\ \text { I } & 31(1) & 25(0) & 18(0) & 11(1) & 5(0) & 2(2) \\ \text { II } & 338(6) & 287(21) & 195(20) & 108(21) & 43(7) & 11(3) \\ \text { III } & 322(8) & 279(26) & 191(29) & 98(20) & 36(8) & 6(1) \\ \text { IV } & 124(14) & 88(17) & 50(10) & 28(12) & 8(2) & 2(1)\end{array}$

Subtypes

D)

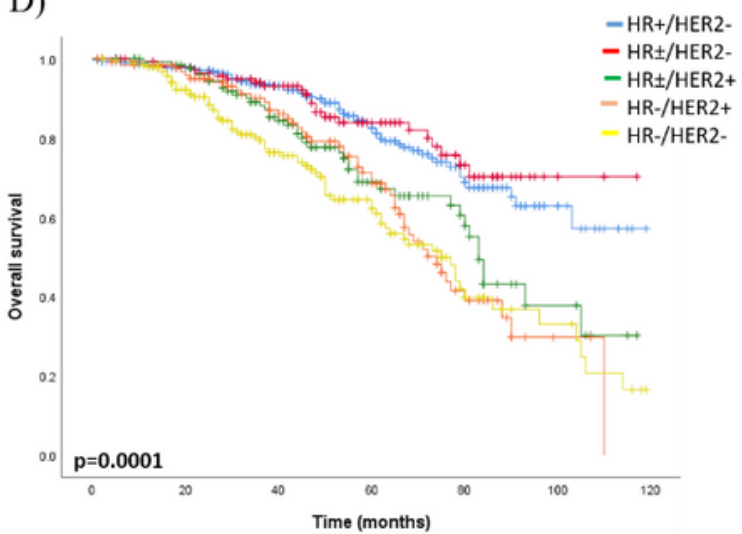

$\begin{array}{lcccccc}\text { No. at risk (events) } & 0 & 20 & 40 & 60 & 80 & 100 \\ \text { HR+/HER2- } & 312(7) & 267(13) & 190(18) & 101(16) & 36(4) & 8(1) \\ \text { HR } \pm \text { HER2- } & 142(3) & 118(6) & 79(8) & 42(5) & 16(1) & 2(0) \\ \text { HR } \pm / H E R 2+ & 130(2) & 113(15) & 71(13) & 34(4) & 18(7) & 4(1) \\ \text { HR-/HER2+ } & 121(4) & 103(10) & 74(13) & 43(17) & 11(3) & 2(1) \\ \text { HR-/HER2- } & 181(14) & 135(23) & 81(13) & 45(15) & 16(3) & 7(4)\end{array}$

Figure 1

Overall survival according to relevant clinical and demographic characteristics. (A) Age; (B) clinical stage at diagnosis; (C) histologic grade; and (D) breast cancer subtypes RH +/HER-(Luminal A), RH \pm /HER2-, $\mathrm{RH} \pm /$ HER2+, RH-/HER2+ (HER2+), RH-/ HER2-(Triple negative).

\section{Supplementary Files}


This is a list of supplementary files associated with this preprint. Click to download.

- SuppFig.png 